\title{
Medium Frequency Transformer Leakage Inductance Modeling and Experimental Ver- ification
}

M. Mogorovic and D. Dujic

This material is posted here with permission of the IEEE. Such permission of the IEEE does not in any way imply IEEE endorsement of any of EPFL's products or services. Internal or personal use of this material is permitted. However, permission to reprint / republish this material for advertising or promotional purposes or for creating new collective works for resale or redistribution must be obtained from the IEEE by writing to pubs-permissions@ieee. org. By choosing to view this document, you agree to all provisions of the copyright laws protecting it. 


\title{
Medium Frequency Transformer Leakage Inductance Modeling and Experimental Verification
}

\author{
Marko Mogorovic and Drazen Dujic \\ Power Electronics Laboratory - PEL \\ École Polytechnique Fédérale de Lausanne - EPFL \\ Station 11, CH-1015 Lausanne \\ marko.mogorovic@epfl.ch,drazen.dujic@epfl.ch
}

\begin{abstract}
This paper provides detailed analytical modeling and finite elements method (FEM) analysis of the medium frequency transformer (MFT) leakage inductance, as one of the key design factors governing the operation of galvanically isolated power electronics converters. Precise leakage inductance modeling in design stage is especially important for converter topologies based on resonant conversion where MFT is a part of a resonant circuit. A comprehensive analytical model that takes into account both the geometric and frequency effects on the given MFT leakage inductance is generated based on the transformer physical structure, thus allowing for optimization of the MFT design with targeted equivalent circuit leakage inductance reference. The derived models are benchmarked to the measurement results on the developed MFT prototype.
\end{abstract}

\section{NOMENCLATURE}

$A_{p} \quad$ - MFT area product (measure of size)

$P_{n} \quad$ - MFT nominal power

$K_{f} \quad$ - Excitation waveform coefficient

$K_{u}$ - Window utilization coefficient

$B_{m} \quad$ - Peak flux density

$J \quad$ - Current density

$f_{s} \quad$ - Switching frequency

$W_{m}$ - Energy of the leakage magnetic field

$L_{\sigma} \quad$ - Total leakage inductance

$I \quad$ - MFT RMS current

$\sigma \quad$ - Electric conductivity

$N_{s} \quad$ - Total number of strands within the winding

$N_{s v} \quad$ - Total number of strands vertically

$N_{s h} \quad$ - Total number of strands horizontally

$m$ - Number of equivalent foil layers

$N_{1} \quad$ - Number of primary winding turns

$l_{w} \quad$ - Winding mean length turn

\section{INTRODUCTION}

Research in directions of novel galvanically isolated conversion topologies, referred to in literature as power electronic transformers PETs [1] or solid state transformers SSTs [2], has been intensified recently. While generally, these are multistage conversion topologies, their inner stages are mostly based either on resonant or dual active bridge (DAB) converter topologies which both feature the MFT as a central key component for both the galvanic isolation and input-output voltage level matching, as displayed in Fig. 1.

The main driver of this research is the substantial reduction in size of the transformer that can be achieved with the increase of the operating frequency, as illustrated with approximate relation (1). As can be seen, while transformer size is affected by various design degrees of the freedom, the electrical parameters of the transformer are not directly included into consideration.

$$
A_{p} \approx \frac{P_{n}}{K_{f} K_{u} B_{m} J f_{s}}
$$

In case of line frequency transformers (LFTs) for high power, magnetizing inductance is usually designed to be very high, while the leakage inductance is normally very low, or sufficiently high to keep short circuit currents below a desired level. With the increase of the operating frequency and use of different power electronic converters, these design rules change and require much higher design fidelity.

The choice of the converter topology has direct implications on the required MFT electrical specification. Namely, MFTs operating in resonant converter topologies typically have a much lower leakage inductance reference compared to their DAB counterparts. This represents a limiting constraint when it comes to MFT design for high and medium voltage which, depending on the dielectric material, typically require large inter-winding dielectric spacing, to facilitate the high blocking

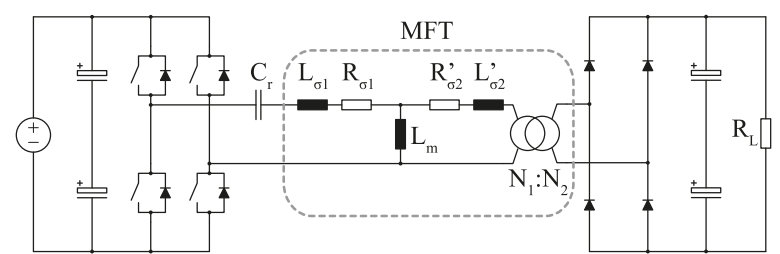

(a)

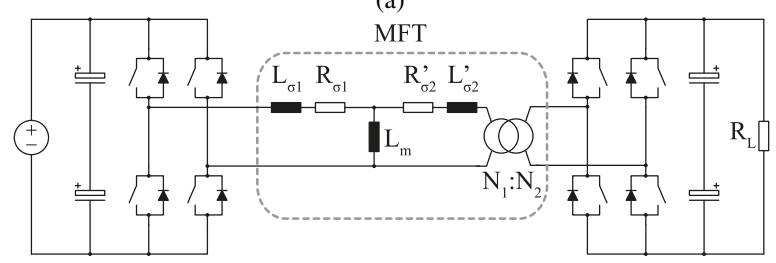

(b)

Fig. 1. DC-DC converter topologies commonly used within SSTs: (a) Resonant converter (b) DAB converter 
voltage requirements, where the leakage field energy is the most concentrated.

Furthermore, the operation of the MFT within the power electronic converter on high frequencies and with nonsinusoidal excitation is coupled with additional modeling and design challenges concerning the electric, dielectric and thermal characteristics [3]. Accurate estimation of the MFT electrical parameters is paramount for the correct design and operation of any converter [4], [5]. This is especially true for topologies based on the resonant conversion where the transformer equivalent circuit is a part of the resonant circuit. In addition to this, the characteristically very low leakage inductance reference makes the required modeling precision even higher and harder to match.

This paper provides a detailed electromagnetic FEM analysis, derivation and experimental verification of the comprehensive analytical leakage inductance model, based on the Dowell's frequency dependent leakage model for foil wingdings [4] and Rogowski approximation [6], capable of accurately estimating the total MFT leakage inductance and its geometric and frequency dependency. This model is suitable for the design optimization purposes [7] due to its simplicity and low computational cost, thus allowing to include the leakage inductance estimation into the design optimization procedure.

\section{MFT DESIGN OPTIMIZATION}

Modeling of the occurring physical phenomena is a necessary step of any design campaign. An example of a brute force MFT design optimization algorithm developed in [7] is displayed in Fig. 2. As can be seen, in the structure of this algorithm, the corresponding models hold the central place, allowing for estimation of the characteristics of each MFT design variation. Therefore, the overall execution speed of the algorithm is heavily dependent on the computation cost of every utilized model and the accuracy of the whole

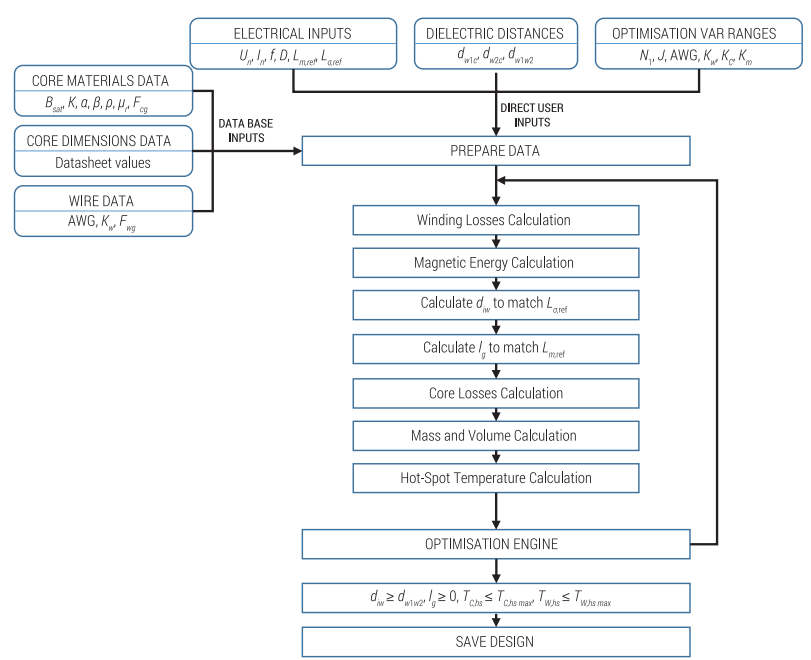

Fig. 2. Brute force MFT design optimization algorithm methodology is only as good as that of the utilized models. This conclusion is general for all optimization schemes, regardless of the level of sophistication. Moreover, the majority of formal mathematical optimization schemes would even imply an additional constraint in terms of model convexity in order to claim with certainty that the found solution represents the global optimum.

The most detailed and accurate class of models, FEM models, are not suitable for overall design optimization considering many variables within a multi-physical design space (e.g. due to high computational cost, problems with convergence), but are rather useful for verification of the models and the final design, as an intermediary step before prototyping.

To that end, this paper presents the derivation of a comprehensive computationally non-intensive, yet sufficiently accurate, analytical leakage inductance model, that takes into account both the frequency and geometry effects, as one of the key MFT parameters.

\section{Analytical Leakage Inductance Model}

Most of the effort focused on analytical modeling of the leakage inductance documented in the literature is based on the integration of the magnetic energy resulting from the leakage magnetic field within the core window area [4]-[6] according to $(2)$.

$$
W_{m}=\frac{L_{\sigma} I^{2}}{2}=\frac{1}{2} \mu_{0} \int_{V}|H|^{2} d V
$$

Most of this work resides on an assumption that the effect of the radial magnetic field on the energy density within the window area and therefore the leakage inductance is negligible. In other words, the magnetic field within the window area is assumed only to have the axial component. This is a sufficiently accurate approximation for most of the simple winding constructions such as one shown in Fig. 3a. The traditional analytical leakage estimation techniques developed for LFTs [6] mostly focus on capturing the winding geometry effect on the leakage. On the other hand, in case of power electronic aplications, the operating frequencies are typically high and therefore the effect of the frequency on the leakage inductance has to be accurately modeled as well. The majority of the of the developed frequency dependent leakage models that can be found in the literature [4], [5], [8] are based on the Dowell's frequency dependent leakage inductance model for foil windings [9] and its generalization via the porosity factor.

\section{A. Application of Dowell's Model on Litz Wire Winding}

In case of a square litz wire winding such as shown in Fig. 3a, circular strands can be substituted with square strands, as shown in Fig. 3b with equivalent width according to (3).

$$
d_{e q}=d \sqrt{\frac{\pi}{4}}
$$

According to Dowell, the winding with square strands from Fig. $3 \mathrm{~b}$ can further be substituted with the equivalent 


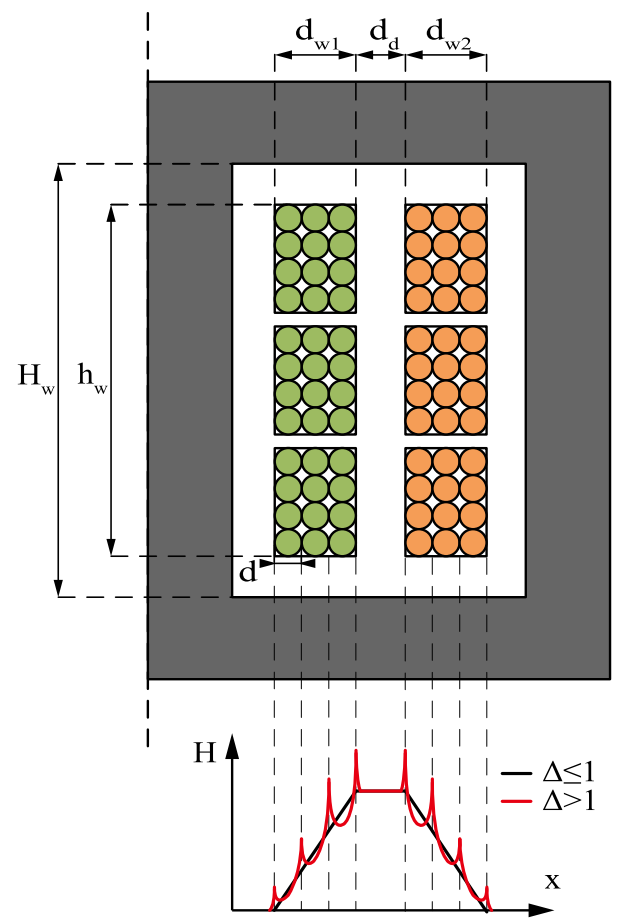

(a)

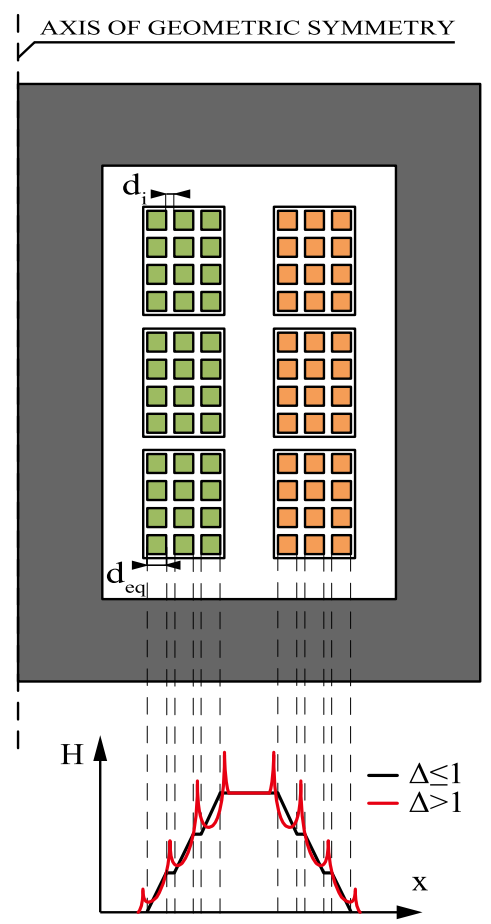

(b)

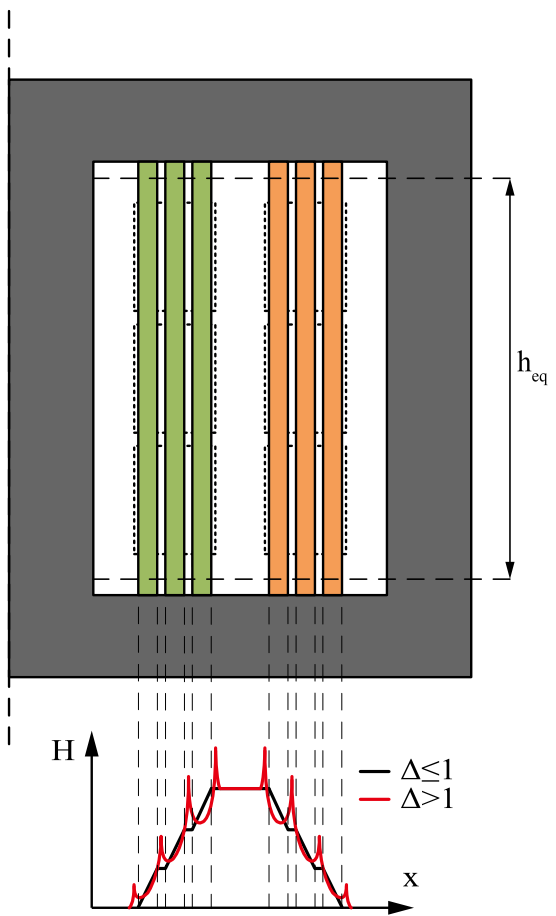

(c)

Fig. 3. Winding equivalence for the leakage inductance estimation with corresponding estimated leakage magnetic field distribution: (a) Original square-profile litz wire winding (b) Equivalent square-profile litz wire winding with square strands (c) Equivalent foil winding

foil winding whose width is equal to the equivalent square strand width and whose height is the same as the height of the core window area, as shown in Fig. 3c. This last equivalence changes the winding copper cross section area and an equivalent porosity factor must be taken into account to ensure the same overall winding DC conductivity according to (4).

$$
\sigma^{\prime}=\sigma \eta
$$

where the porosity factor is calculated as given in (5).

$$
\eta=\frac{N_{s v} d_{e q}}{H_{w}}
$$

However, the cross-section of any real litz wire does not form a matrix with perfectly aligned strands, as shown in Fig. 3a. The positioning of the strands is rather chaotic and non-constant along the wire length due to the necessary transpositions and twisting of litz strands and groups of strands. This is especially true for square litz wires, featuring high number of very thin strands, as they undergo an additional pressing process in order to shape them into their final rectangular profile.

Therefore, determining the number of litz layers within the windings in horizontal $\left(N_{s h}\right)$ and vertical $\left(N_{s v}\right)$ direction is not straightforward. However, for the purposes of this model, the equivalent numbers of strands can be generated using an assumption that they have to follow the same geometric proportion as that of the total winding cross-section profile as given in (6).

$$
K_{w}=\frac{h_{w}}{d_{w}}
$$

With this assumption, the equivalent numbers of strands of a perfectly aligned and spatially distributed litz wire winding equivalence, as displayed in Fig. 3a, in horizontal and vertical direction, can be calculated as shown in (7) and (8), respectively.

$$
\begin{gathered}
N_{s h}=\sqrt{\frac{N_{s}}{K_{w}}} \\
N_{s v}=\sqrt{K_{w} N_{s}}
\end{gathered}
$$

This equivalence provides the necessary parameters of the equivalent foil winding for implementation of Dowell's frequency dependent leakage inductance model displayed in Fig. 3c, namely the number of equivalent foil winding layers $\left(m=N_{s h}\right)$ and the porosity factor, as given in (5).

The application of Dowell's model on the equivalent foil winding results in a closed form analytical expression for leakage inductance estimation given in (9). 


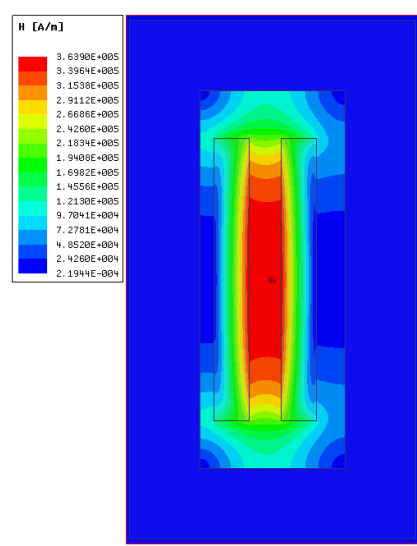

(a)

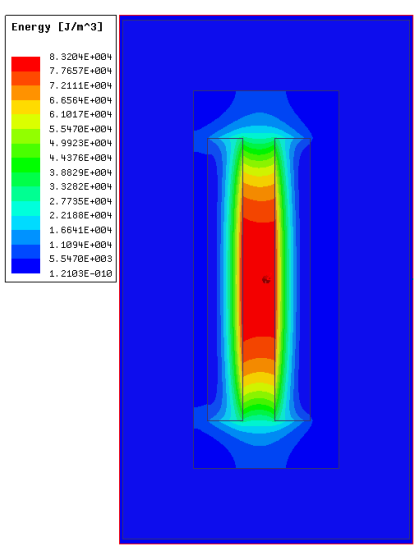

(b)

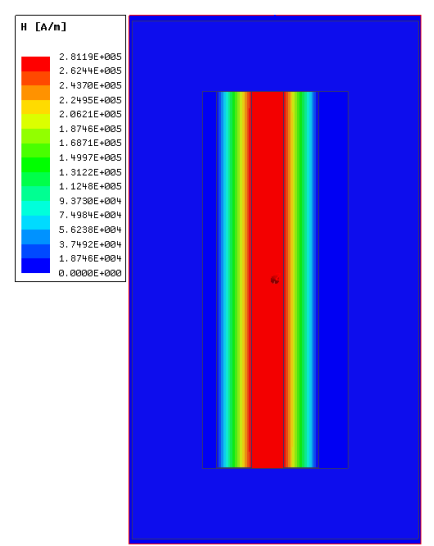

(c)

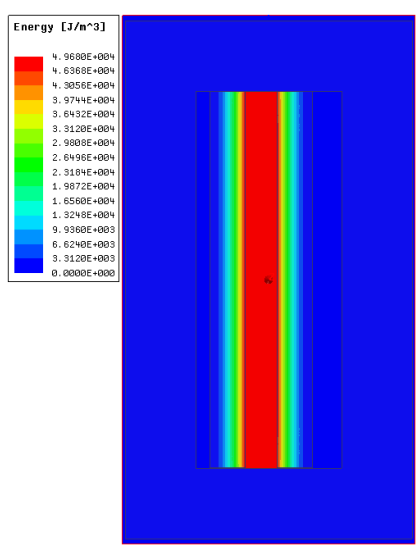

(d)

Fig. 4. 2D FEM magnetostatic simulations: (a) Magnetic field distribution within the actual geometry (b) Magnetic energy distribution within the actual geometry (c) Magnetic field distribution within the Dowell equivalent geometry (d) Magnetic energy distribution within the Dowell equivalent geometry

$$
\begin{gathered}
L_{\sigma}=N_{1}^{2} \mu_{0} \frac{l_{w}}{H_{w}} \underbrace{\underbrace{d_{w 1 e q} m_{w 1}}_{\begin{array}{c}
\text { Portion due to magnetic energy within } \\
\text { the inter-winding dielectric volume }
\end{array}} F_{w 1}+\frac{d_{w 2 e q} m_{w 2}}{3} F_{w 2}}_{\begin{array}{c}
\text { Frequency dependent portion due to the magnetic } \\
\text { energy within the copper volume of the windings }
\end{array}} \\
+\underbrace{d_{w 1 i} \frac{\left(m_{w 1}-1\right)\left(2 m_{w 1}-1\right)}{6 m_{w 1}}}_{\begin{array}{c}
\text { Portion due to magnetic energy within the } \\
\text { inter-layer dielectric of the primary winding }
\end{array}} \\
+\underbrace{d_{w 2 i} \frac{\left(m_{w 2}-1\right)\left(2 m_{w 2}-1\right)}{6 m_{w 2}}}_{\begin{array}{c}
\text { Portion due to magnetic energy within the } \\
\text { inter-layer dielectric of the secondary winding }
\end{array}}]
\end{gathered}
$$

The frequency dependent portion of the leakage magnetic field, within the copper volume of the primary and secondary winding, is taken into account via Dowell's frequency dependent factor (10), applied on the equivalent foil winding as shown in Fig. 3c.

$$
F_{w}=\frac{1}{2 m^{2} \Delta}\left[\left(4 m^{2}-1\right) \varphi_{1}-2\left(m^{2}-1\right) \varphi_{2}\right]
$$

where

$$
\varphi_{1}=\frac{\sinh (2 \Delta)-\sin (2 \Delta)}{\cosh (2 \Delta)-\cos (2 \Delta)}
$$

and

$$
\varphi_{2}=\frac{\sinh (\Delta)-\sin (\Delta)}{\cosh (\Delta)-\cos (\Delta)}
$$

and equivalent penetration ratio and skin depth are given in (13) and (14), respectively.

$$
\Delta=\frac{d_{e q}}{\delta}
$$

and

$$
\delta=\sqrt{\frac{1}{\pi \mu_{0} \sigma^{\prime} f_{s}}}
$$

\section{B. FEM Analysis}

To test the validity of the Dowell's equivalence and the accuracy of the estimation, magnetostatic 2D FEM simulations are performed on the actual transformer cross-section geometry (generated utilizing the MFT design optimization procedure described in [7], as illustrated in Fig. 2) and equivalent Dowell geometry as a DC boundary condition, as displayed in Fig. 4, respectively. The FEM estimate of the DC leakage inductance of the actual MFT geometry is $L_{l_{f e m(a b)}}=8.25 \mu \mathrm{H}$ while the one of the Dowell's equivalence is $L_{l_{f e m(c d)}}=7.12 \mu \mathrm{H}$. These results show that already at DC, Dowell's model has a deviation of roughly $13.7 \%$ due to the inaccurate geometry equivalence.

As the only parameter that changes with this equivalence is the winding height $\left(h_{w}\right)$ or, in other words, the proportion between the winding height and core window height $\left(H_{w}\right)$, additional parametric sweep is performed with the given 2D FEM models, varying the winding height in range from $40 \%$ to $100 \%$ of the core window height. These results are compared with the leakage inductance estimation of the Dowell's model at zero frequency (DC) and shown in Fig. 5.

One can notice that the estimation error increases substantially with the decrease of the mentioned proportion. This result is intuitively clear as the change of the geometry introduced by Dowell's equivalence is higher as the proportion of the winding height compared to core window height is smaller. Thus, the estimation of Dowell's model has reasonable accuracy only for geometries where the winding to core window height proportion is close to $100 \%$.

\section{Hybrid Leakage Inductance Model}

In traditional LFT design, the effect of winding geometry on the transformer leakage inductance is taken into account via a correction factor, Rogowski factor [6], that takes into account the uneven geometric magnetic field distribution by correcting 
the equivalent length (winding height) of the magnetic flux path through the air.

The main idea of the hybrid model proposed in this paper is to use this corrected equivalent magnetic field path length for winding equivalence in Dowell's frequency dependent model instead of the total core window height, thus including the geometry implications into the described framework. This equivalent winding height can be calculated from the original winding height with (15).

$$
h_{e q}=\frac{h_{w}}{K_{R}}
$$

where

$$
K_{R}=1-\frac{1-e^{-\pi h_{w} /\left(d_{w 1}+d_{d}+d_{w 2}\right)}}{\pi h_{w} /\left(d_{w 1}+d_{d}+d_{w 2}\right)}
$$

The correction of the model is simply applied by substitution of $H_{w}$ with the equivalent height $h_{e q}$ from (15) in (4) and (9), thus generating a hybrid leakage inductance model that accurately captures both the frequency and geometry effects.

The leakage inductance estimations of the developed hybrid model, for various winding to core window height ratios at
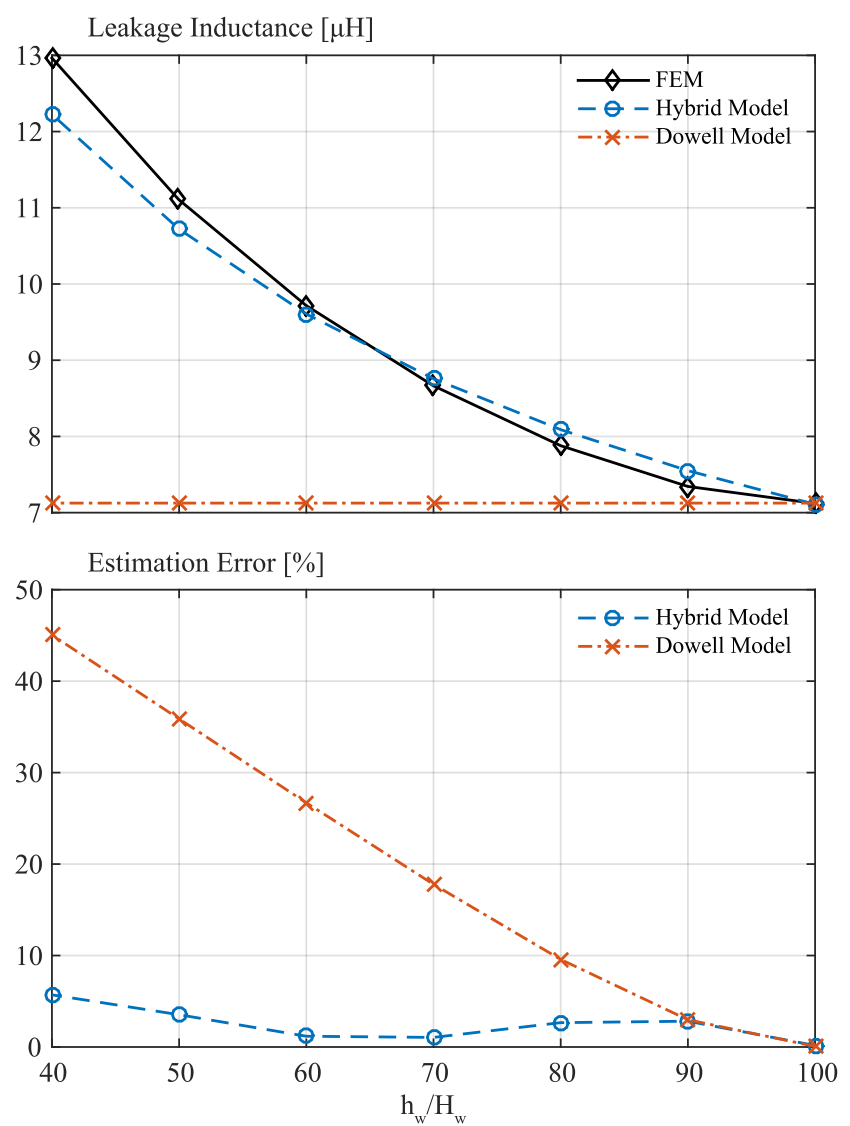

Fig. 5. Top plot: Magnetostatic FEM simulation leakage inductance estimation (black), estimation with hybrid model at zero frequency (blue dashed line), estimation with Dowell model at zero frequency (red dash-dot line); Bottom plot: DC leakage inductance estimation error of the Hybrid (blue dashed line) and Dowell (red dash-dot line) model, referred to the FEM results zero frequency (DC), are compared with the FEM simulations and Dowell's model in Fig. 5. It can be seen that the hybrid model estimation is very well correlated with FEM simulation results, with maximum relative error of roughly $6 \%$ in the entire given geometric proportion range, from $40 \%$ to $100 \%$. These results confirm the capability of the developed model to accurately account for the effect of the MFT geometry in a much wider range compared to original Dowell's model.

\section{MFT PROTOTYPE AND EXPERIMENTAL VALIDATION}

For verification purposes, a full scale MFT prototype with electric specifications, as given in Table I, has been realized according to the optimal design technical specifications generated by the MFT optimization algorithm (Fig. 2). The details about the overall optimization methodology and MFT prototype are extensively reported in [7]. The realized $100 \mathrm{~kW}, 10 \mathrm{kHz}$ MFT prototype, connected to the impedance measurement setup consisting of a Bode100 network analyzer is displayed in Fig. 6

TABLE I

N87 SIFERRIT MFT PROTOTYPE ELECTRIC SPECIFICATIONS

\begin{tabular}{|c|c|c|c|c|c|}
\hline$P_{n}$ & $V_{1}$ & $V_{2}$ & $f_{s w}$ & $L_{s p}, L_{s s}$ & $L_{m}$ \\
\hline $100 \mathrm{~kW}$ & $750 \mathrm{~V}$ & $750 \mathrm{~V}$ & $10 \mathrm{kHz}$ & $4.2 \mu \mathrm{H}$ & $750 \mu \mathrm{H}$ \\
\hline
\end{tabular}

The proposed hybrid leakage inductance model has been experimentally verified on the given measurement setup. The measurement results, in form of a frequency sweep in range of $2 \mathrm{kHz}$ to $1 \mathrm{MHz}$, are displayed in Fig. 7 in the top plot, together with the original Dowell and proposed hybrid leakage inductance model estimations. The relative errors of these two models referred to the measurement are displayed in Fig. 7 in the bottom plot. These results show that the hybrid model has a significantly better accuracy (less than $4 \%$ error) compared to the Dowell model (around 18\% error) in the given frequency

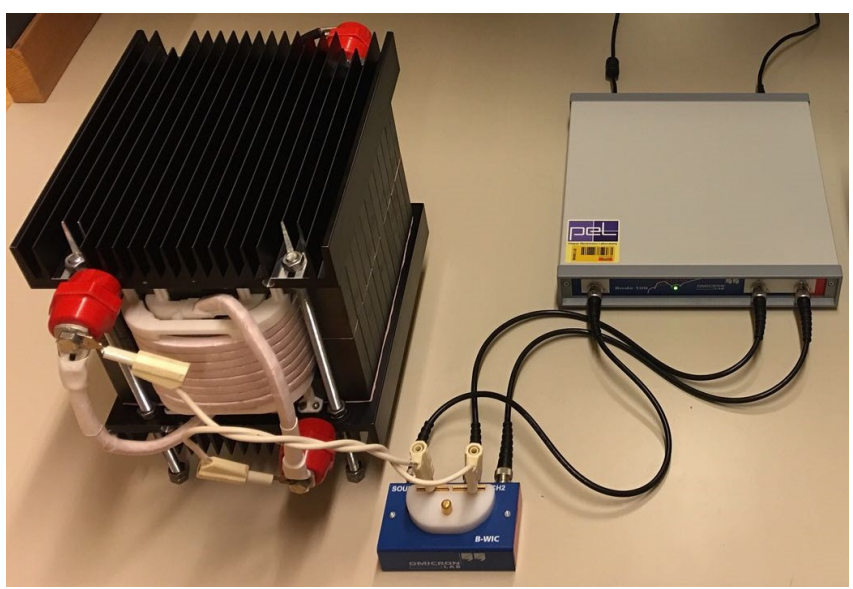

Fig. 6. Measurement of the leakage inductance on the MFT prototype using Boode 100 

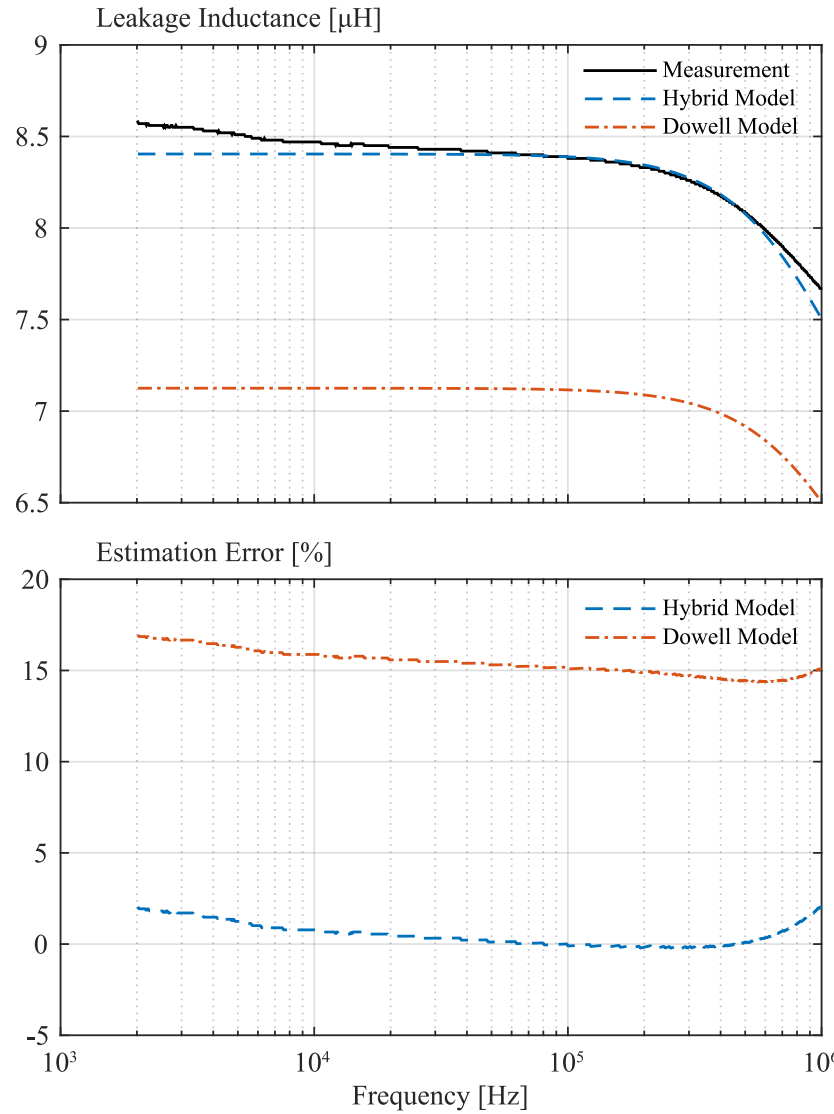

Fig. 7. Top plot: leakage inductance measurement (black), estimation with hybrid model (blue dashed line), estimation with Dowell model (red dashdot line); Bottom plot: leakage inductance estimation error, of the Hybrid (blue dashed line) and Dowell (red dash-dot line) model, referred to the measurement

range which corresponds to the previously elaborated DC leakage estimation error due to the Dowell's equivalence.

\section{CONCLUSION}

Appropriate MFT modeling for design optimization is a complex task. Besides the accuracy requirements, facilitating any optimization scheme implies hard constraints in terms of simplicity and computational cost, thus excluding the possibility of direct application of very sophisticated numeric tools such as FEM. This paper presents a computationally nonintensive, yet sufficiently accurate, analytical leakage inducta- nce model, capable of appropriately accounting for both the frequency and geometric effects.

Details are provided how litz-wire windings can be transformed, while preserving the leakage field distribution, into an appropriate form for the application of the Dowell's frequency dependent leakage inductance model. It has been shown that Dowell's equivalence does not accurately take into account the geometry of the transformer and its applicability is reduced to a very low range of MFT geometries, where winding height is very close to the core window height. A method capable of including the geometry influence into the developed framework in a very simple and elegant way was developed. Namely, it was shown that it is possible to supplement the Dowell's model by simply correcting the winding equivalent height using the Rogowski factor that appropriately takes into account the winding geometry, thus generating the hybrid leakage inductance model that inherits both the accurate frequency and geometric dependency. The FEM simulations and measurement results confirm that the developed hybrid model is drastically more accurate, in a much larger range of MFT geometric proportions, compared to the original Dowell's model, whose applications on different winding types are considered as current state of the art.

\section{REFERENCES}

[1] M. Claessens, D. Dujic, F. Canales, J. K. Steinke, P. Stefanutti, and C. Vetterli, "Traction Transformation: A Power-Electronic Traction Transformer (PETT)," ABB Review, No: 1/12, pp. 11-17, 2012.

[2] J. E. Huber and J. W. Kolar, "Solid-State Transformers: On the Origins and Evolution of Key Concepts," IEEE Ind. Electron. Mag., vol. 10 no. 3, pp. 19-28, Sep. 2016.

[3] M. Mogorovic and D. Dujic, "Thermal modeling and experimental verification of an air cooled medium frequency transformer," in 19th European Conference on Power Electronics and Applications (EPE'17 ECCE Europe), Warsaw, Poland, 2017. (accepted),

[4] I. Villar, "Multiphysical Characterization of Medium-Frequency Power Electronic Transformers," PhD thesis, EPFL Lausanne, Switzerland 2010.

[5] M. Bahmani, "Design and Optimization Considerations of MediumFrequency Power Transformers in High-Power DC-DC Applications," $\mathrm{PhD}$ thesis, Chalmers University of Technology Gothenburg, Sweden, 2016.

[6] S. V. Kulkarni and S. A. Khaparde, Transformer Engineering: Design and Practice. Marcel Dekker, Inc., 2004.

[7] M. Mogorovic and D. Dujic, "Medium frequency transformer design and optimization," in Power Conversion and Intelligent Motion - (PCIM) 2017, Nuremberg, Germany, 2017., pp. 423-430.

[8] A. Van den Bossche and V. C. Valchev, Inductors and Transformers for Power Electronics. Taylor \& Francis, Mar. 2005.

[9] P. L. Dowell, "Effects of eddy currents in transformer windings," Proc. of the Institution of Electrical Engineers, vol. 113, no. 8, pp. 1387-1394, Aug. 1966. 